\title{
Ueber die Spaltung von Polysacchariden durch verchiedene Hefenenzyme.
}

\author{
Von \\ Anusch. Kalanthar.
}

(Aus dem I. Berliner Universitätslaboratorium.)

(Der Redaction zugegangen am 15. August 1898.)

Durch die Versuche von E. Fischer weiss mn, dass nicht allein der Rohrzucker, sondern auch die übrign Polysaccharide erst dann die alkoholische Gährung dura Hefen erleiden, wenn sie zuvor eine hydrolytische Spaltung a Monosaccharide erfahren haben. Den Beweis dafür hat rr, zum Theil in Gemeinschaft mit P. Lindner, durch das Studium der in den Hefen enthaltenen hydrolytischen Enzyme ;eliefert. Seine Beobachtungen betreffen verschiedene Arten vo untergähriger und obergähriger Bierhefe, ferner Milchzucerhefen und Kefirkörner, dann Sacch. Marxianus, Schizo-Saca. octosporus und Monilia candida.

Um den Resultaten eine breitere Basis zu gebn, habe ich auf Veranlassung von Herrn Professor E. Fischer ihnliche Versuche nach denselben Methoden auf folgende Iffearten und Mikroorganismen ausgedehnt:

1. Sechs Weinhefen, und zwar von: Bordeauxwen 1893, Ungarwein Menes 1894, italienischem Wein Bari Italiaa 1893, Rheinweinen (Rauenthaler Berg 1894, Assmannshäusr 1892 und Steinberg 1893).

2. Bierhefen aus Bayern und Rostock.

3. Weissbierhefen von Berlin und Lichtenhain ferner Hefe des Negerbiers Pombe und Logoshefe. 
4. Hefen des russischen Getränkes Kissly-Schtschi.

5. Hefen des armenischen, kefirähnlichen Getränkes Mazun.

Bezüglich der Herkunft derselben bemerke ich Folgendes: Die sechs Weinhefen wurden mir von der oenologischen Anstalt $\mathrm{zu}$ Geisenheim a. Rh. durch freundliche Vermittlung des Herrn Prof. Dr. Wortmann überlassen. Näheres darüber findet man in den Berichten dieser Anstalt. Die meisten übrigen Hefen verdanke ich Herrn Professor Lindner. Sie sind später mit den Nummern bezeichnet, welche sie in der Kulturensammlung des Instituts für Gährungsgewerbe Berlin (Ecke See- und Torfstrasse) führen. Einige Hefen aus Mazun und Kissly-Schtschi habe ich selbst mit freundlicher Unterstützung des Herrn Professor Lindner isolirt. Alle Hefen waren Reinkulturen, aus einer Zelle und in ungehopfter Bierwürze gezüchtet; nur bei den Kulturen von Mazunhefen wurden noch Traubenmost, ferner Traubenzucker oder Rohrzucker unter Zusatz von Pepton und Nährsalzen angewendet. Die Kulturen wurden von der Mutterlauge so, wie E. Fischer es vorgeschrieben hat, durch ein Pukall'sches. Filter getrennt und so lange mit reinem Wasser gewaschen, bis eine Probe Fehling'sche Lösung sich beim Kochen nicht mehr veränderte.

Wie aus der späteren tabellarischen Uebersicht meiner Beobachtungen hervorgeht, wurden die Hefen entweder im frischen, oder im getrockneten Zustand, oder endlich in Form eines wässerigen Auszugs geprüft. Unter frischen Hefen verstehe ich die filtrirten und nur mit Wasser gewaschenen Reinkulturen. Bei allen späteren Versuchen mit denselben kamen folgende Mengenverhältnisse in Anwendung:

$$
\begin{aligned}
& 0,6 \text { gr. frische Hefe, } \\
& 0,6 \text { gr. Polysaccharid, } \\
& 6 \text { ccm. reines Wasser, } \\
& 0,075 \text { gr. Toluol. }
\end{aligned}
$$

Letzteres ist nach dem Vorgang von E. Fischer gewählt, um die alkoholische Gährung zu verhindern.

Die trockenen Hefen waren durch Aufbringen der frischen auf porösen Thon und nachfolgendes dreitägiges Aufbewahren 
an der Luft hergestellt. Die Mengenverhältnisse waren folgende:

$$
\begin{aligned}
& 0,25 \text { gr. trockene Hefe, } \\
& 0,5 \text { gr. Polysaccharid, } \\
& 5 \text { ccm. Wasser, } \\
& 0,075 \text { gr. Toluol. }
\end{aligned}
$$

Der benutzte Auszug war stets durch 15stündiges Auslaugen von 1 Theil trockener Hefe mit 15 Theilen Wasser bei $30^{\circ}$ bereitet. Auf 10 Theile Auszug wurde 1 Theil Polysaccharid und 0,1 Theil Toluol angewandt. Das Erwärmen geschah im Brutschrank, und die Mischungen waren in zugeschmolzenen Reagensgläsern enthalten.

Von Polysacchariden kamen zur Anwendung Rohrzucker, Maltose, Milchzucker, Melibiose, Trehalose, Melitriose (Raffinose), Melicitose und endlich das a-Methylglucosid.

Die Menge der entstardenen Monosaccharide wurde bei Rohrzucker, Trehalose, Melitriose, Melicitose und $\alpha$-Methylglucosid durch Titration mit Fehling'scher Lösung bestimmt und daraus die Quantität der zersetzten Polysaccharide berechnet. Bei Maltose, Milchzucker und Melibiose, welche selbst die Fehling'sche Lösung reduziren, wurde das Monosaccharid durch Phenylhydrazin erkannt. Leider gestattet das Reagens keine genaue quantitative Bestimmung. Um aber eine annähernde Vorstellung von dem Grade der Zersetzung zu geben, habe ich in den Tabellen die Menge des aus dem entstandenen Monosaccharid gebildeten Phenylosazons angegeben. Die Zahlen beziehen sich auf 0.6 gr. bezw. 0.5 gr. Polysaccharid.

In Tabelle I ist das Verhalten der Weinhefen, in Tabelle II dasjenige der Bierhefen und in Tabelle III dasjenige der Mazunhefen geschildert.

Zur weiteren Erläuterung derselben erwähne ich Folgendes:

Rohrzucker und Raffinose sind von fast allen Hefen gleich stark gespalten worden. Besonders stark war die Spaltung bei der Weinhefe Bari Italiana $(99,75 \%)$ und der Brennereipresshefe Róstock (100\%). Wie die Tabelle I zeigt, ist bei Rohrzucker die Thätigkeit des Invertins durch eine Temperatur von $60^{\circ}$ nicht aufgehoben worden. 


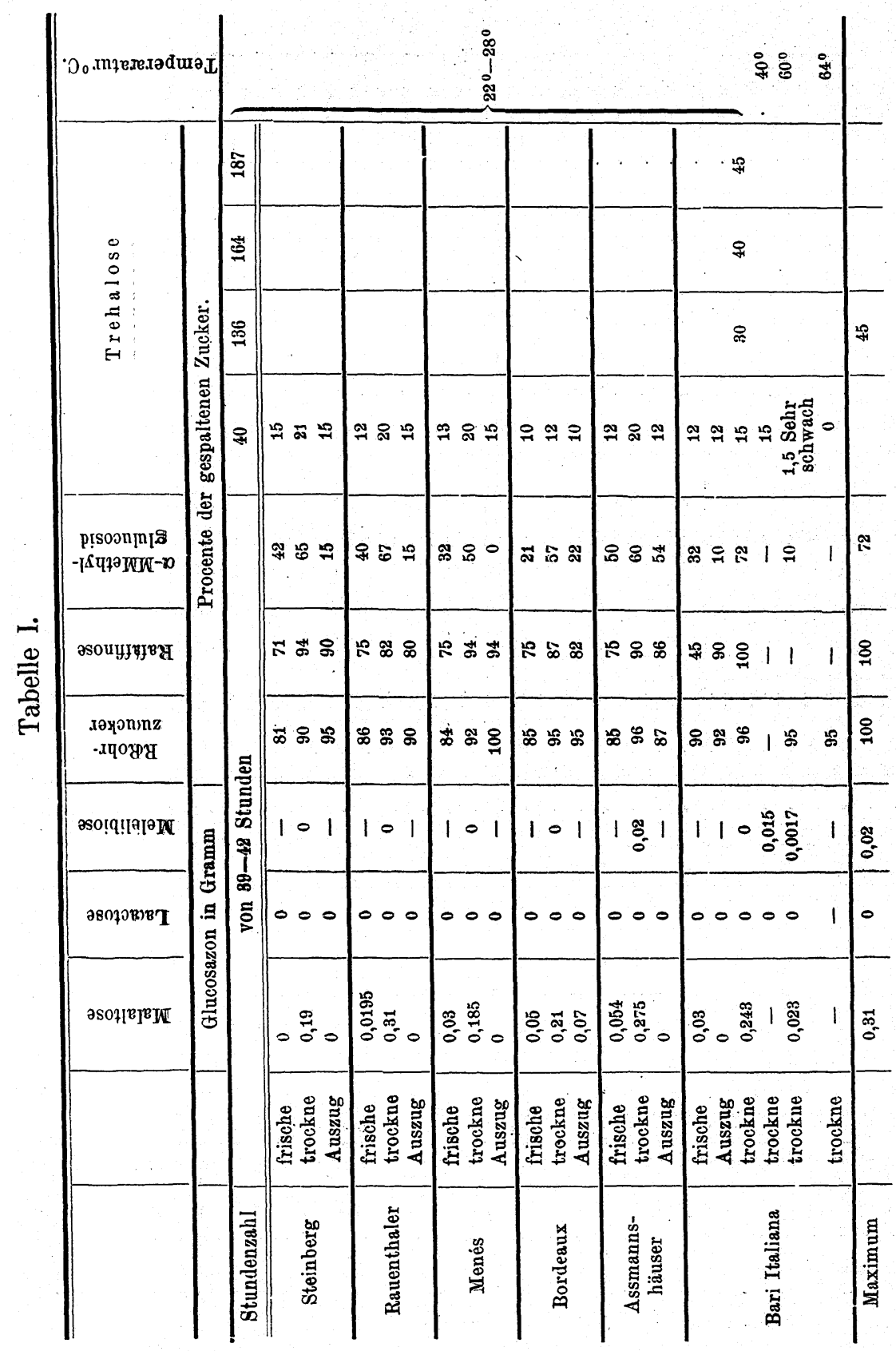


Tabelle II.

\begin{tabular}{|c|c|c|c|c|c|c|c|c|}
\hline . & & 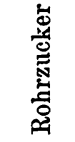 & 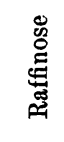 & 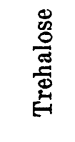 & 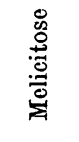 & 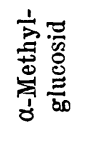 & 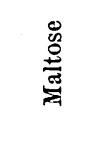 & 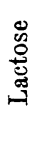 \\
\hline & \multicolumn{6}{|c|}{ Procente der gespaltenen Zucker } & \multicolumn{2}{|c|}{ Osazon in Gr. } \\
\hline \multirow{3}{*}{ Rostocker } & frische & 100 & 75 & 10 & - & 一 & 0,21 & 0 \\
\hline & trockne & 100 & 86 & 37,5 & - & 50 & $0,20 ̄ 8$ & 0 \\
\hline & Auszug & 100 & 100 & 17,5 & - & 15 & 0 & 0 \\
\hline \multirow{3}{*}{ Bayerische } & frische & 80 & 50 & 12,5 & 12,5 & 30 & 0,14 & 0 \\
\hline & trockne & 90 & 80 & 35 & $\check{5} 0$ & 80 & 0,29 & 0 \\
\hline & Auszug & 90 & 80 & 10 & 40 & 55 & 0,205 & 0 \\
\hline \multirow{3}{*}{ Weissbier } & frische & 60 & 30 & 5 & 20 & 22,5 & 0,03 & 0 \\
\hline & trockne & 90 & 72 & 10 & 30 & 65 & 0,20 & 0 \\
\hline & Auszug & 90 & 75 & 5 & 30 & 50 & 0,15 & 0 \\
\hline \multirow{3}{*}{$\begin{array}{l}\text { Kissly- } \\
\text { Schtschi }\end{array}$} & frische & 45 & 48 & 0 & 10 & 0 & 0 & 0 \\
\hline & trockne & 90 & 90 & 20 & 22,5 & 2 & 0,01 & 0 \\
\hline & Auszug & 90 & 90 & 10 & 25 . & 0 & 0 & 0 \\
\hline \multirow{3}{*}{$\begin{array}{l}\text { Lichten- } \\
\text { hainer }\end{array}$} & frische & 75 & 38 & 8 & 20 & 25 & 0,03 & 0 \\
\hline & trockne & 90 & 70 & 10 & 30 & 50 & 0,15 & 0 \\
\hline & Auszug & 90 & $7 \check{~}$ & 5 & 2,5 & 30 & - & 0 \\
\hline \multirow{3}{*}{ Pombe } & frische & 25 & 37 & 0 & 12,5 & 20 & - & 0 \\
\hline & trockne & 80 & 75 & 5 & 45 & 37,5 & 0,328 & 0 \\
\hline & Auszug & 80 & 75 & 0 & 60 & 30 & 0,25 & 0 \\
\hline \multirow{3}{*}{ Logos } & frische & - & - & - & - & - & 0,0345 & 0 \\
\hline & trockne & 90 & 75 & 25 & 30 & 65 & 0,15 & 0 \\
\hline & Auszug & 90 & 75 & 0 & 30 & 65 & 0,03 & 0 \\
\hline
\end{tabular}

Temperatur: 24,50; Zeitdauer: 39-42 Stunden. 


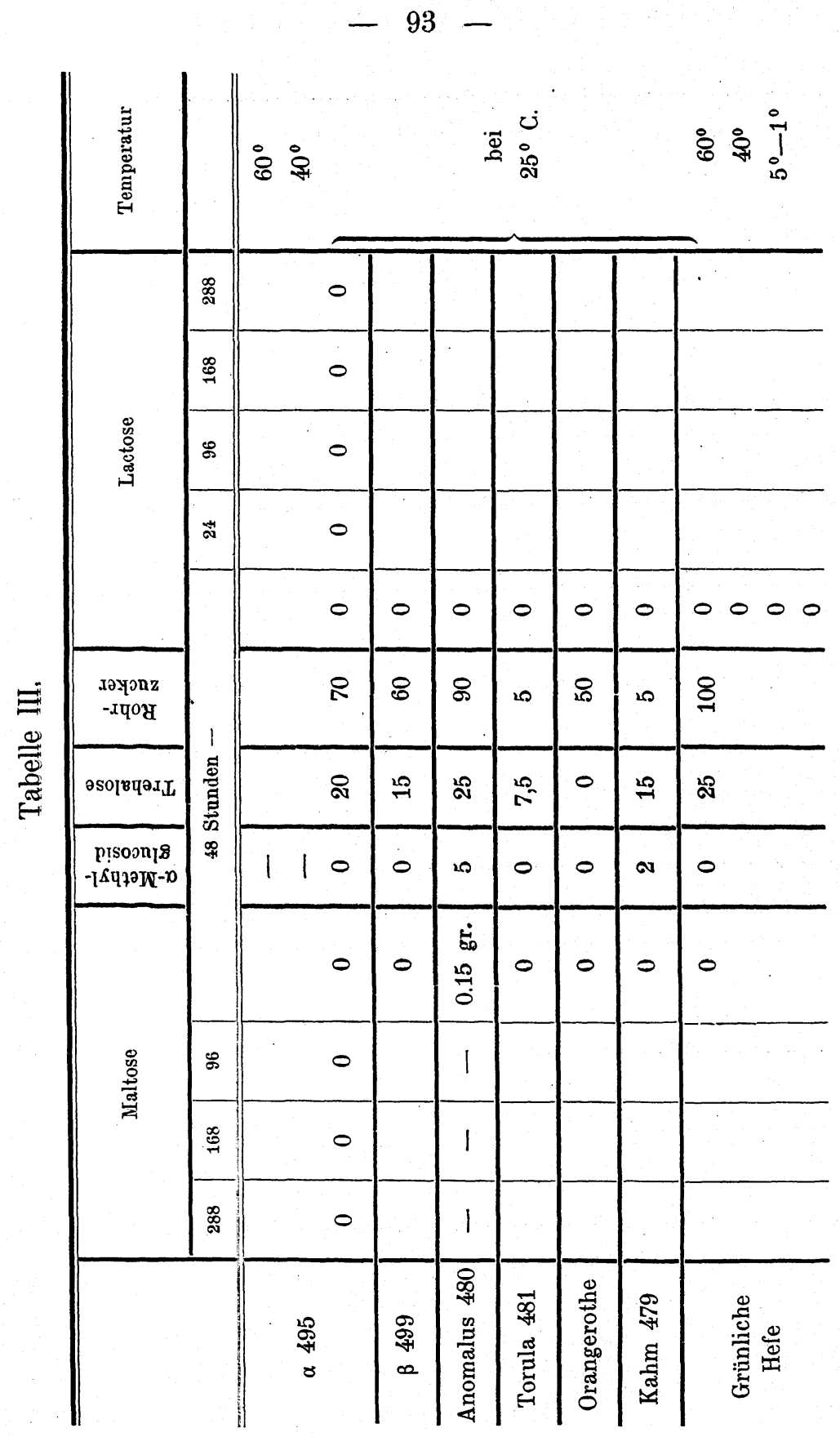


Für Maltose und $\alpha$-Methylglucosid ist als angreifendes Enzym Hefenmaltase (oder Hefenglucase) angenommen. Für a-Methylglucosid betrug das Maximum der Spaltung bei Weinhefen $72 \%$ (s. trockene Barihefe Tab. I), für Brennereihefen $80 \%$ (s. Bayerische Hefe Tab. II). Die gespaltene Maltose wurde durch Glucosazon bestimmt. Es wurden auf 0,6 gr. Maltose 0,825 gr. Phenylhydrazin und 0,825 gr. 50\% ige Essigsäure verwendet. Das Ganze wurde 1/2 Stunden auf dem Wasserbade erhitzt, das Phenylglucosazon von dem löslichen Maltosazon durch kochendes Wasser getrennt und gewogen. Das Maximum der Spaltung für Maltose beträgt bei trocknen Hefen für 0,5 gr. Maltose 0,31 gr. (s. Rauenthaler Tab. I) und 0,328 gr. (s. Pombe Tab. II).

Maltose und $\alpha$-Methylglucosid verhielten sich in den meisten Fällen sehr ähnlich; wo z. B. die Maltosespaltung abgeschwächt war, da galt dasselbe auch für $\alpha$-Methylglucosid. Bei Anwendung von Kissly-Schtschihefen zeigte Maltose minimale Quantitäten $(0,01$ gr. Osazon) und $\alpha$-Methylglucosid war auch nur schwach gespalten (kaum 2\%). Bei bayerischer Hefe erhielt ich neben $80 \%$ a-Methylglucosidspaltung auch eine erhebliche Hydrolyse der Maltose $(0,29$ gr. Osazon Tab. II). Aber trotz aller dieser Uebereinstimmungen liessen sich auch Verschiedenheiten feststellen. So wurde z. B. von den Hefenauszügen von Steinberg, Bari Italiana, Rauenthaler, Assmannshäuser Maltose nicht in nachweisbarer Menge gespalten, wohl aher $\alpha$-Methylglucosid. Hierzu ist aber zu bemerken, dass die Erkennung von kleinen Mengen Traubenzucker neben viel Maltose durch Phenylhydrazin Schwierigkeiten bietet.

Die Versuche mit Lactose zeigen keine positiven Resultate in Bezug auf Hydrolyse, wie auch die Temperatur und Zeitdauer gewählt wurden.

Für Melibiose fielen die Resultate bei den verschiedenen Temperaturen nicht gleich aus. Bei trockener Bari ItalianaHefe fand bei einer Temperatur von $40^{\circ}$ eine deutliche Hydrolyse statt, bei niedrigeren Wärmegraden $\left(25^{\circ}-30^{\circ}\right)$ dagegen war eine solche niemals zu konstatiren. Assmannshäuser Rheinweinhefe ergab ein interessantes Resultat, da durch sie schon 
bei $25^{\circ}$ Spaltung erzielt wurde. Alle mit den übrigen Weinhefen meiner Versuchsreihe angestellten Spaltungsproben ergaben bei einer Temperatur von $24,5^{\circ}$ kein positives Resultat.

Ich habe diese seltene und nur schwer rein herstellbare Zuckerart nach der Methode von Scheibler und Mittelmeier ${ }^{1}$ ) aus Melitriose bereitet und auf ihre Reinheit vermittelst des Osazons geprüft. Bekanntlich hat Melibiose dieselben Componenten wie Milchzucker (d-Glucose und d-Galactose), aber gegenüber den Hefenenzymen verhalten sie sich ganz verschieden. Bei $40^{\circ}$ und $60^{\circ}$ ergab sich für Melibiose Spaltung, für Lactose aber nicht; das Optimum lag bei etwa $40^{\circ}$.

Die Trehalose ist bis jetzt als schwer spaltbare Zuckerart bekannt. Meine Hefen haben, mit Ausnahme der orangerothen Mazunhefe, ziemlich erhebliche spaltende Wirkung ausgeübt. Auch hier wirken frische Hefen immer schwächer als trockene. Alle angewandten Hefen enthalten also wirksames Enzym. Bei $24^{0}-25^{\circ}$ wurden innerhalb 42 Stunden durch frische Weinhefen $15 \%$ und durch Brennereihefen 12\% gespalten. Andere Frischhefen wirken gar nicht oder sehr schwach. Bei Verwendung trockenen Materials dagegen wurden im Maximum durch Weinhefen 21\%, durch Brennereihefen $37,5 \%$ und durch andere Hefen $10 \%$ gespalten. Mit steigender Temperatur nimmt die Intensität der Spaltung nicht merklich zu, wohl aber ist die Menge des gespaltenen Zuckers von der Dauer des Versuches abhängig. Denn bei Bari Italiana waren bei $26^{\circ}$

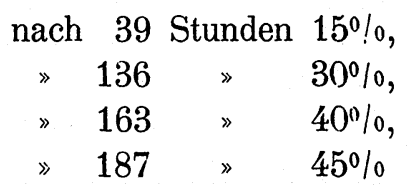

gespalten. E. Fischer ${ }^{2}$ ) hat Spaltung der Trehalose durch Bierhefen erzielt, und zwar wurden von trockener Hefe $20 \%$ hydrolysirt, während der wässerige Auszug keine Spaltung bewirkte. Das Resultat stimmt mit dem meinigen für Pombe und Logos überein; aber bei meinen Weinhefen und allen übrigen ist Hydrolyse auch durch wässerigen Hefenauszug bewirkt worden.

1) Ber. d. deutsch. chem. Gesellsch. 22, 1678 u. 23, 1438.

2) Ber. d. deutsch. chem. Gesellsch. 28, 1432. 
Bourquelot ${ }^{1}$ ) hat bereits eine Spaltung der Trehalose durch Aspargillus niger und durch Grünmalz beobachtet und das wirksame Enzym Trehalase genannt. Das in meinen Weinhefen wirksame Enzym stimmt mit Bourquelot's Trehalase darin überein, dass auch hier die Wirksamkeitsgrenze bei etwa $64^{0}$ liegt. Ob letztere identisch ist mit dem in den Weinhefen enthaltenen Enzym, bleibt noch festzustellen.

Auf Melicitose habe ich alle Hefen der Tabelle II, mit Ausnahme der Rostocker, einwirken lassen. Bei Logos wurde frische Hefe nicht verwendet. Die Resultate waren in allen geprüften Fällen positiv. Das Maximum der Spaltung wurde durch Pombe erreicht, und zwar mit $60 \%$ bei wässerigem Auszug.

\section{Beschreibung der einzelnen Hefen.}

Wie schon erwähnt, wurden mehrere der untersuchten Hefen aus dem Milchpräparat Mazun, welches ich aus meiner Heimath Armenien bezogen habe, isolirt. Da dasselbe dem wissenschaftlichen Publikum kaum bekannt sein dürfte, so will ich seine Eigenschaften und seine Gewinnung beschreiben. Mazun ist kefirähnlich, hat aber einen eigenartigen, angenehmen Geschmack, wodurch es sich von Kefir und anderer saurer Milch unterscheidet. Das beste Mazun wird aus Büffel- oder Ziegenmilch bereitet, besonders in den Städten verwendet man aber auch ein Präparat, welches aus gewöhrlicher Kuhmilch hergestellt ist. Seine Hauptbedeutung liegt in der Verwerthung zur Butterbereitung. Der grösste Theil der gebräuchlichen und exportirten armenischen Butter ist aus Mazun gewonnen. Bei Armeniern und Kurden zieht man diese Butter der aus süsser Sahne hergestellten vor, weil sie angenehmer schmeckt und ein Aroma besitzt, welches ohne Zweifel durch die im Mazun befindlichen Organismen verursacht wird.

Ausser bei der Bereitung der Butter hat das Mazun noch andere grosse Bedeutung im täglichen wirthschaftlichen Leben. Man benützt es zur Anrichtung von Milchspeisen oder, besonders zur Sommerszeit, als Getränk, indem man es theils

1) Bull. soc. mycol. 9, 189. 
unmittelbar mit Jöffeln geniesst, theils mit Wasser vermischt. So wird es namentlich in heissen Gegenden und in Ebenen verwendet, wo wegen des sehr miasmatischen und Fieber verursachenden Klimas Wassergenuss schädlich ist und Gebrauch von Wein oder anderen alkoholischen Getränken wegen der grossen Hitze bei der Feldarbeit nachtheilig wirken kann. Nach der Butterbereitung bleibt saure Buttermilch zurück; letztere wird ebenfalls im Sommer als kühlendes Getränk genossen oder zu anderen Produkten verarbeitet. Wenn die Buttermilch in einem Thongefäss ruhig steht, so setzt sie sich nach 1-4 Tagen als eine käsige Masse ab, über welcher sich die klare Molke befindet. Diese Quargmasse wird in einem Sack ausgepresst und dann Than genannt. Letzterer wird mit Mehl versetzt, in Stückchen geschnitten und an der Sonne getrocknet, um so für die langen Winter, wie sie im türkischen Armenien vorkommen, aufbewahrt zu werden. In der Provinz Wan wird dieser Tschora-Than (trockener Than) mit Spinat und Reis, unter Zusatz von Pfeffermünze und anderen einheimischen Gewürzen, unter fortwährendem Umrühren gekocht. Es ist ein besonders beliebtes Gericht und heisst dann Than-apur.

Man bereitet Mazun in folgender Weise: Nachdem die Keime der Milch durch Kochen abgetötet sind, wird sie bis zur Blutwärme abgekühlt und mit einem Theil alten Mazuns vermengt. Das letztere wird, ehe es unter die Milch gemischt wird, mit so viel warmer Milch oder kaltem Wasser versetzt, bis es dünnflüssig ist. Das Ganze bringt man dann sofort in einen Topf, der mit einem dicken Tuch umhüllt wird. Hierauf lässt man es im Sommer an einem geschützten Ort stehen, während es im Winter anfänglich an einer warmen Stelle aufbewahrt werden muss. Um zum Genuss steiferes Mazun zu haben, muss man es dann einige Zeit an einen kühlen Ort stellen.

Die Art der Zubereitung hat mich zu der Vermuthung geführt, dass das Mazun seine Entstehung im Wesentlichen der Thätigkeit von Mikroorganismen verdankt. Diese Vermuthung hat sich bestätigt, denn unter dem Mikroskop erkennt man verschiedene Organismen: Hefen, ziemlich grosse Bacillen, Mikrokokken und Schimmelpilze, die ich alle in Reinkultur besitze.

Hoppe-Seyler's Zeitschrift f. physiol. Chemie, XXVI. 
Die Hefen sind in 9 Arten vertreten, von denen Herr Prof. Lindner vier isolirt hat. Bacillen sind zwei von mir isolirt, der eine aus frischem Mazun, der andere aus Tschora-Than. Eine Mikrokokkus-Species hat Herr Dr. O. Emmerling freundlichst in Reinkultur gezogen. ${ }^{1}$ ) Eine zweite, kleinere ist von mir isolirt worden, die aber möglicherweise mit den ersten identisch sein kann.

Von anderen Pilzen fand ich Oidium lactis und eine Mucorspecies, vermuthlich auch noch einen Aspargillus.

Von den neun Mazunhefen habe ich sieben genauer untersucht. Sie wachsen sowohl auf Bierwürzegelatine als auch auf Lactosegelatine vorzüglich, wenn auch auf letzterer merklich langsamer.

Ich gehe nunmehr zur Beschreibung der von mir speziell untersuchten Hefen über. Ihre morphologische Diagnose ist theilweise von Herrn Prof. P. Lindner freundlichst ausgeführt und mir mitgetheilt worden.

1. Eine Anomalusart erregt wie $\mathrm{S}$. anomalus belgicus in Würze keine Gährung und wächst Mycoderma ähnlich. Der Rand der vom 12. I. 98 bis 26. I. 98 gewachsenen und $1 \mathrm{~cm}$. breit gewordenen Kolonie war rein weiss, während die übrige Fläche einen schwach röthlichbraunen Farbenton angenommen hatte. Sie verflüssigt Gelatine und bildet hutförmige Sporen. Unter Nr. 479 der Berliner Sammlung eingeordnet. (Isolirt von Lindner.)

2. Eine Anomalusart, welche in Würze gährt und angenehm riechenden Fruchtäther entwickelt, nach Bildung einer dünnen Kahmhaut. Wächst auf Bierwürzegelatine in flachen, soliden Kolonien mit monotoner, graugelblicher, matter Oberfläche. Rand mehr grau und schwach concentrisch geschichtet; später treten weisse, mehlig-trockene Zacken auf. Bildet grosse, hutförmige Sporen wie Nr. 479, besonders reichlich im Wassertropfen. Verhält sich Polysacchariden gegenüber wie gewöhnliche Weinhefen, enthält kein Lactase, zeigt schwache $\alpha$-Methylglucosid- $(5 \%)$ und Maltosespaltung. Nr. 481. (Isolirt von Lindner.)

1) Vergl. Centralbl. f. Bacterialogie 1898, Mai. 
3. Torula-Art. Mehr gewölbte Kolonien bildend, ebenfalls mit matter, schwach rosagelber Oberfläche; enthält Trehalase und Invertin. Maltose, $\alpha$-Methylglucosid und Lactose bleiben unberührt. Nr. 480. (Isolirt von Lindner.)

4. Orangerothe Riesenkolonien mit concentrischen Leisten, siegelförmiges Aussehen. In Bierwürze, Traubenmost, Rohrzucker-Lactosenährlösungen keine Gährung. In Tröpfchenkulturen in Bierwürze Zelle mit schleimigem Hof; später reihenweise Gruppirung der Zellen. Wächst in Lactosenährlösung, Molken, Milch, 2\% Chinasäure spärlich, in 4\% Chinasäurenährlösungen ausserordentlich schwach; auf Chinasäureagar (1\% Chinasäure, 2\% Pepton, 3\% Agar-Agar) wächst sie anfangs roth, verliert allmählich den Farbstoff und zeigt nur hier und da an der Oberfläche rothe Tupfen. Enthält nur Invertin; denn Rohrzucker wurde stark gespalten, während alle anderen Zuckerarten unberührt blieben. Kann vielleicht zur Reingewinnung des Invertins dienen. In Traubenzucker unangenehmer Geschmack. In Molkengelatine gibt sie schwache, einfache Stichkultur, gibt in der Tiefe die rothe Färbung ganz aut. Besonders auffallend ist, dass sie keine Trehalase enthält, der einzige Fall unter meinen 20 verschiedenen Hefen. Nr. 497. (Isolirt von mir.)

5. a-Mazunhefe. Wächst fast genau wie Hefe Saaz. Der wellige Rand concentrisch geschichtet, die in der Mitte liegende, muldenförmige Vertiefung mit Wülsten ausgefüllt. Wächst sehr schnell auf Pflaumendecoct, Most, Bierwürze; vergährt Traubenmost und Rohrzucker. Der Traubenzucker nimmt nach der Gährung einen eigenartigen, milchsauren, sehr an Mazun erinnernden Geschmack an. Spaltet Rohrzucker und Trehalose; ist gegen Lactose völlig wirkungslos (selbst beim Zerreiben mit Glaspulver), mit oder ohne Toluolzusatz bei verschiedenen Temperaturen und verchiedener Zeitdauer. Nr. 495. (Isolirt von mir.)

6. Grünliche Mazunhefe. Die Riesenkolonien vom 16. XII. 97 bis 26. I. 98 waren flach ausgebreitet $(2,5 \mathrm{~cm}$. breit), mit wenigen radialen Linien. Oberfächlich matt grünlichgrau. Mit der Zeit steigt die mittlere Partie wie ein flaches Plateau auf, nimmt mehr und mehr pfirsichblüthrothe Farbe an, und schliess- 
lich sinkt die ganze Kolonie in die Tiefe. Vergährt sehr schwach Traubenzucker und Traubenmost, wächst gut auf Pflaumendococtgelatine und Bierwürzegelatine. Traubenzucker schmeckt nach der Gährung mazunsauer. Spaltet Rohrzucker $(100 \%)$ und Trehalose $(25 \%)$. Gegen Lactose und Maltose und auch gegen $\alpha$-Methylglucosid wirkungslos, ebenso wie a-Mazunhefe. Nr. 496. (Isolirt von mir.)

7. Kahmhefe. Riesenkolonien kräftig wachsend, dabei locker, schwammig, etwas crèmefarben. Gelatine nicht verflüssigend. Hydrolysirende Wirkung noch nicht geprüft. Nr. 498.

8. $\beta$-Mazunhefe. Flache, einfache Kolonien, mit matter, bräunlichgrauer Oberfläche. Im Impfstich in Molkengelatine Zerklüftung der Gelatine. Röthliche Färbung an der Berührungsstelle mit der Luft. Vergährt Most sehr schwach, spaltet Rohrzucker und Trehalose. Nach der Gährung milchsauer schmeckend. Nr. 499. (Isolirt von mir.)

9. Ebenso wie Nr. 499. Vergährt Molken, welche nach der Gährung wie Mazun-Buttermilch schmecken. Spaltung noch. nicht genau untersucht. Nr. 500. (Isolirt von Lindner.)

In Molkengelatine-Stichkulturen (nach Lindner) bilden seitlich ausstrahlende Stiche Nr. 479, 498, 495; 480 sendet beim Stich vereinzelt horizontale, fächerförmige Blätter in die Gelatine. 481 und 497 geben schwache, einfache Stichkulturen, 499 und 500 unter der Oberfläche pfirsichblüthrothe Färbung der Gelatine.

Mir scheint, dass a (495), $\beta$ (499), die grünliche Hefe (496), ferner Nr. 500 und Anomalus (480) specifische Bestandtheile des Mazuns sind, weil der sauere Geschmack und das Aroma an Mazun erinnert. Es ist wahrscheinlich, dass die Lactose durch die Schizomyceten und die säurebildenden Hefen gespalten wird, und dass dann diese, an ein saures Substrat gewöhnte Hefen die Alkoholgährung verursachen.

Die Brennereipresshefen sind untereinander ziemlich ähnlich und alle obergährig. Näheres findet man in der Litteratur. ${ }^{1}$ )

1) P. Lindner, Mikroskopische Betriebskontrole. 
Die Weinhefen verhalten sich gegenüber Polysacchariden ebenso wie die gewöhnlichen Bierhefen. Sie besitzen dieselben Enzyme.

Pombehefe wurde aus dem Negerhirsebier isolirt. Sie ist eine Warmhefe und gibt bei hoher Vergährung geringen Ernteertrag mit glattem Bodensatz. Die Riesenkolonien wachsen langsam, sind kompakt, bilden einen steilen Kegel und zeigen gar keine Gliederung. Die gährende Flüssigkeit wird verhältnissmässig stark sauer. Bei gewöhnlicher Temperatur vergährt sie Dextrin sehr langsam, bei einer Temperatur von etwa $25^{\circ} \mathrm{R}$. aber energisch. Pombehefe ist ein Schizosaccharomycet, der sich also durch Theilung und nicht durch Sprossung vermehrt. Sehr intensiv wird Maltose gespalten, Trehalose dagegen sehr schwach, und bei Versuchen mit frischer Hefe und Auszug war die Hydrolyse bei letzterer kaum nachweisbar. Sehr starke Hydrolyse erleidet auch Melicitose.

Die Logoshefe wurde von van Laer aus einem brasilianischen Biere isolirt. Sie ist auch eine Warm- und Klumphefe. Sie wächst sehr charakteristisch in Riesenkolonien, ähnlich wie S. Pasteurianus III, vergährt bei gewöhnlicher Temperatur Dextrin und bewirkt neben starker $\alpha$-Methylglucosidspaltung schwache Spaltung der Maltose. Der wässerige Auszug griff Trehalose und Lactose nicht an.

Schliesslich wurde aus dem russischen Getränk KisslySchtschi eine Hefe isolirt. Diese bildet sehr fest anhaftende Bodensätze und vergährt die Bierwürze nur wenig. Die Bierwürze erinnert nach der Gährung sehr an Kissly-Schtschi (schwach milchsauer und weissbierähnlich). Die Riesenkolonien sind zartgekräuselt und von grauweisser Farbe. Die einzelnen Zellen haben glatten Plasmainhalt und sind zum Theik klein, an S. exiguus erinnernd. Die trockene Hefe erzeugt starke Spaltung des Rohrzuckers, nur sehr schwache Hydrolyse von Maltose, a-Methylglucosid und Trehalose. Frische Hefe wirkt überhaupt nicht, der wässerige Auszug wirkt auf Maltose und a-Methylglucosid nicht. Diese Hefe enthält, wie alle anderen, Invertase. 